\title{
Polystyrene Core-Silica Shell Particles with Defined Nano Architectures as a Versatile Platform for Suspension Array Technology
}

\author{
Dominik Sarma, Kornelia Gawlitza and Knut Rurack* \\ Chemical and Optical Sensing Division 1.9 \\ Bundesanstalt für Materialforschung und -prüfung (BAM) \\ Richard-Willstätter-Str. 11, D-12489 Berlin, Germany \\ knut.rurack@bam.de
}

\section{Supporting Information}

\section{Content}

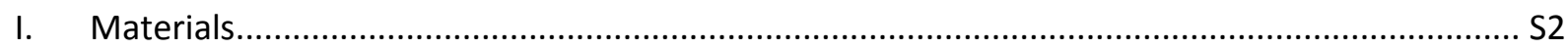

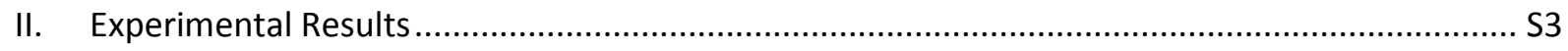

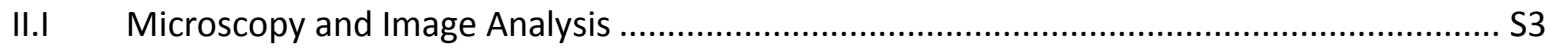

II.II Nitrogen adsorption/desorption and particle calculations ............................................. S6

II.III Sedimentation, chemical surface characterization and assay performance ........................ S7

II.IV Correlation plots for all cytometric measurements ........................................................... S9 


\section{Materials}

Poly(vinylpyrrolidone) (PVP10 with 10 kDa, PVP40 with 40 kDa and PVP360 with 360 kDa, Sigma), styrene (ReagentPlus, $<99 \%$, Sigma) basic alumina $\left(\mathrm{Al}_{2} \mathrm{O}_{3}\right.$, Brockmann I, Acros) and 4,4'-azobis(4-cyanovaleric acid) (ACVA, MP Biomedicals) were used for the PVP-coated polystyrene core synthesis. Ammonium hydroxide (32\% in water, Merck) and tetraethoxyorthosilicate (TEOS, <99\%, Merck) were used for the silica coating and hydrochloric acid ( $\mathrm{HCl}, 37 \%$, Merck), fluorescein isothiocyanate isomer I (FITC, $\geq 90 \%$, Sigma), aminopropyltriethoxysilane (APTES, 97\%, ABCR) and succinic anhydride (97\%, Sigma) were used for surface modification. FAM- (6-carboxyfluorescein) labeled oligonucleotides (5'- C6 Amino-TTT CAT CCA TC -6-FAM -3') were purchased from Metabion (Steinkirchen, Germany) and coupled with $\mathrm{N}$-(3-dimethylaminopropyl)- $\mathrm{N}$ ethylcarbodiimide hydrochloride (EDC, BioXtra grade, Sigma). We used MES buffer (10 mM, $\mathrm{pH}=4.5,2-(N$-morpholino)ethanesulfonic acid $)$ and TRIS buffer $(10 \mathrm{mM}, \mathrm{pH}=8.0$, TRIS/SDS (0.05 \%SDS), TRIS/TE (1mM TE), tris(hydroxymethyl)aminomethane (TRIS), sodium dodecyl sulfate (SDS), ethylenediaminetetraacetic acid (TE)) for coupling and washing, respectively. Hybridization to c-DNA- (5'-C6- $\mathrm{NH}_{2}$-TTT ATG TCG TTT GCT GTA-3', Metabion GmbH, Germany) modified particles was performed with FAM-labeled t-DNA (3'TAC AGC AAA CGA CAT-5', Metabion GmbH, Germany) in TMAC (tetramethyl ammonium chloride) buffer, which was prepared according to Luminex Corp. protocols. Phosphate Buffer (PB, 0.1M, pH = 7.4) was used for cytometric analysis. Methanol (Alfa Aesar, Germany), ethanol (abs. 99\% and 96\%, ChemSolute) and water of MilliQ grade (BAM) were used as solvents and for washing. All reagents were used without any further treatment. 


\section{Experimental Results}

\section{II.I Microscopy and Image Analysis}

a)

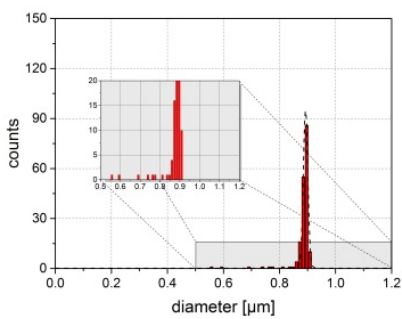

d)

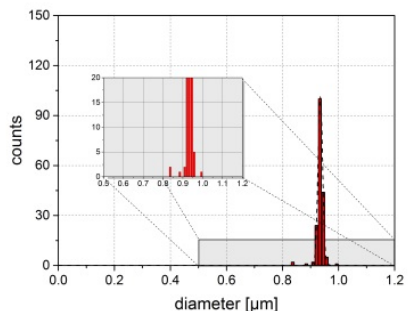

b)

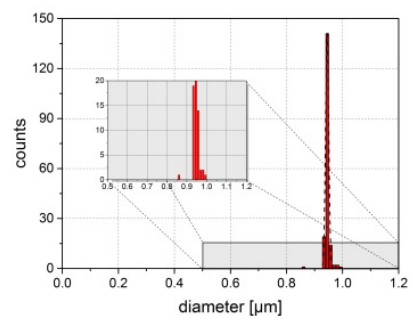

e)

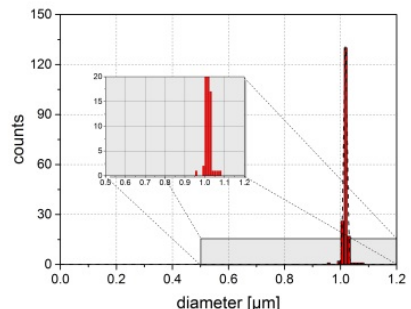

c)

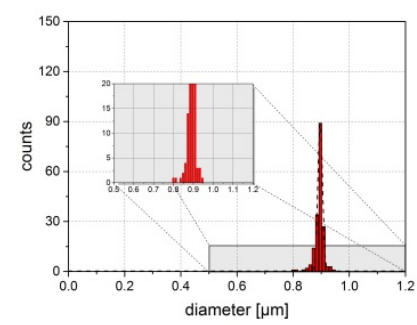

f)

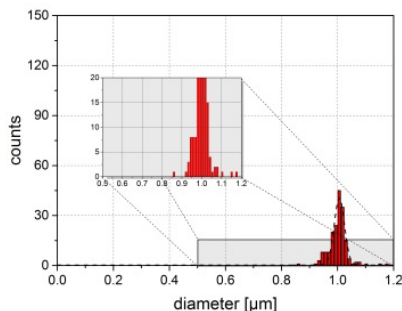

Figure S1. Particle size distributions of polymeric cores $(a, b, c)$ and CS particles (d,e,f) derived from T-SEM image analysis with ImageJ software using Gaussian fits of main peak: (a) PS10, (b) PS40, (c) PS360, (d) SiO2@PS10, (e) $\mathrm{SiO}_{2} @ P S 40,(f) \mathrm{SiO}_{2} @ P S 360$. Only in the case of PS10, a minor population (ca. 5\%) of considerably smaller particles was traceable in the T-SEM overview (Figure 1a, Figure S1a), which is reflected by the highest $c_{v}$ of 4.6\% for this particle batch.
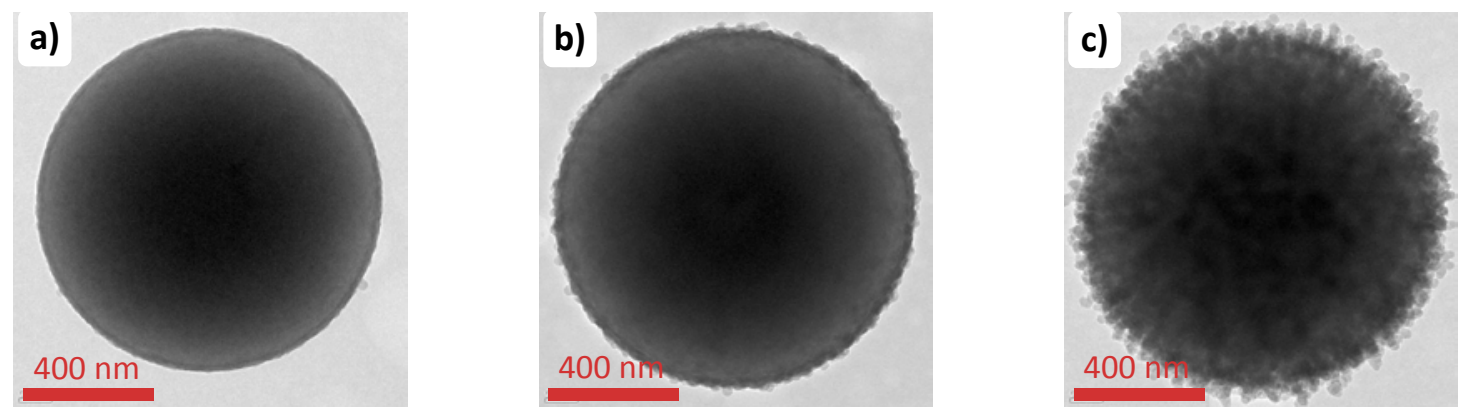

Figure S2: Representative TEM images taken from the SiO ${ }_{2} @ P S$ particles; (a) $\mathrm{SiO}_{2} @ P S 10,(b) \mathrm{SiO}_{2} @ P S 40,(c)$ $\mathrm{SiO}_{2} @ \mathrm{PS} 360$. 

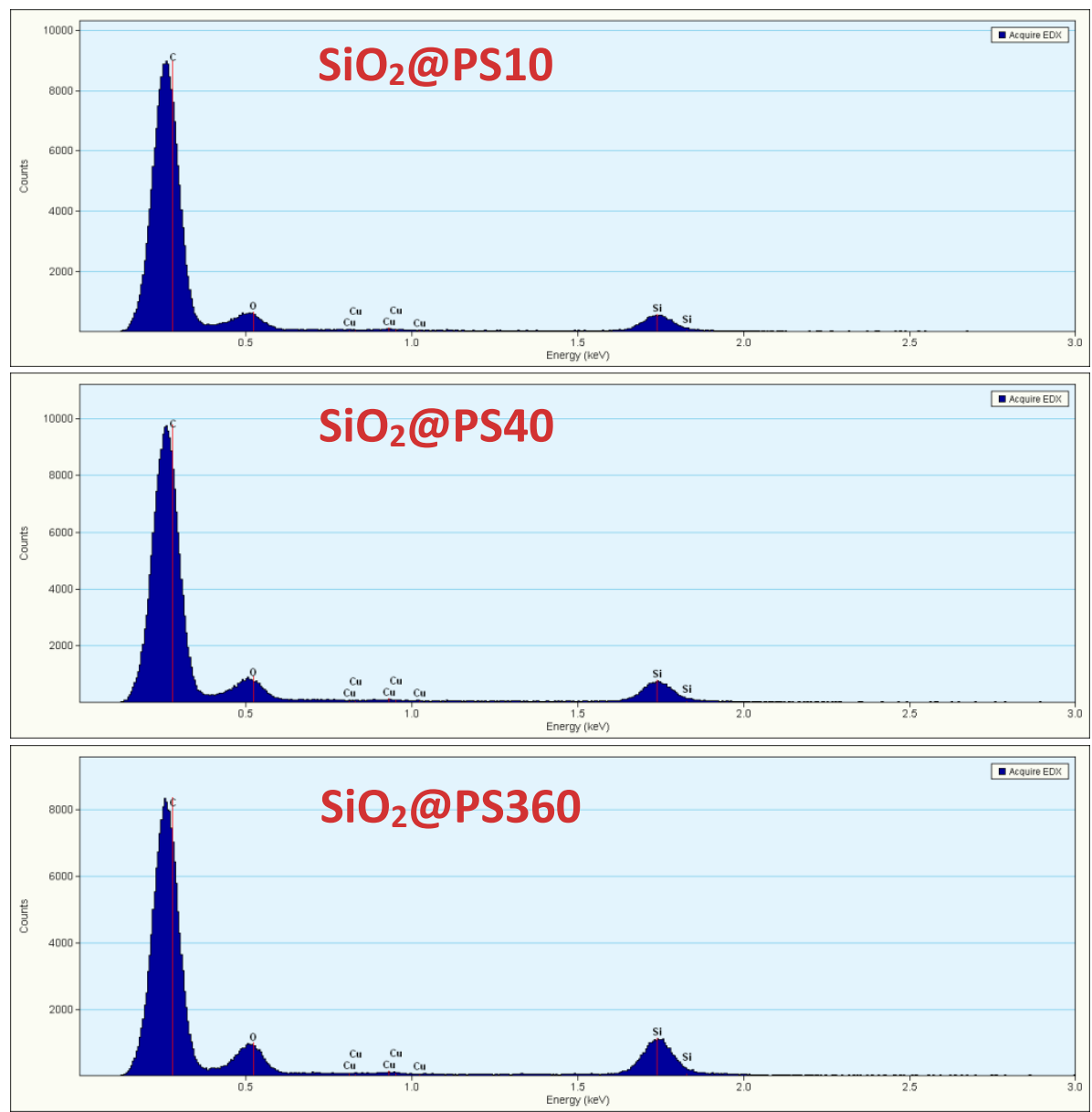

Figure S3: EDX measurements of representative particles.
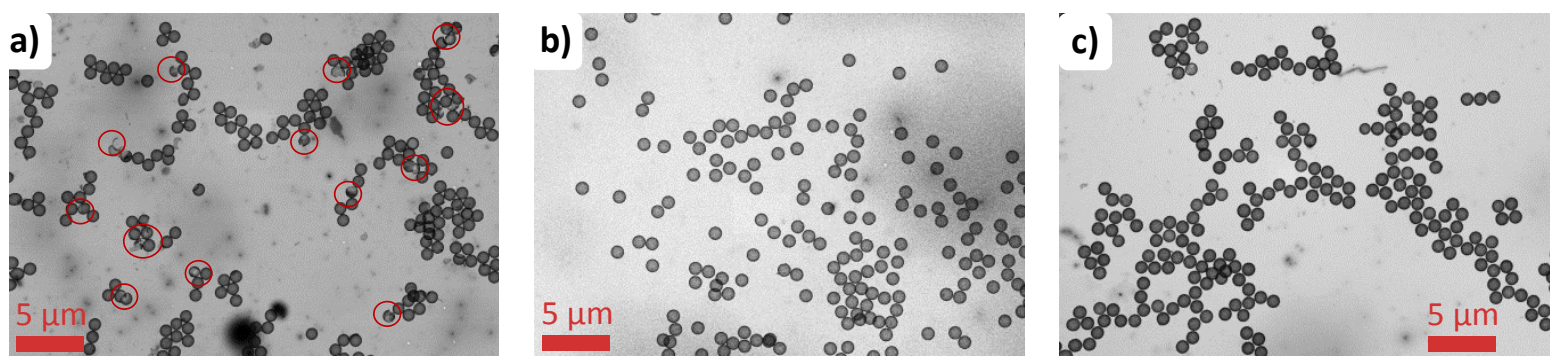

Figure S4: SEM images of SHMs after thermal removal of the polymeric core from (a) $\mathrm{SiO}_{2} @ \mathrm{PS} 10,(\mathrm{~b})$ $\mathrm{SiO}_{2} @ P S 40$ and (c) SiO $2 @ P S 360$ precursors. 
Table S1: Zeta potential (ZP) and size determinations of PS beads as well as $\mathrm{SiO}_{2} \mathrm{NPs}$ formed during the coating process.

\begin{tabular}{c|ccccc} 
& $\begin{array}{c}\mathrm{ZP} \text { in coating } \\
\text { medium }(\mathrm{mV})\end{array}$ & $\begin{array}{c}\mathrm{ZP} \text { in water } \\
(\mathrm{mV})\end{array}$ & $\begin{array}{c}\mathrm{R}_{\mathrm{h}} \text { of } \mathrm{SiO}_{2} \\
\mathrm{NP}^{\mathrm{a}}(\mathrm{nm})\end{array}$ & $\mathrm{PDI}^{\mathrm{b}}$ & $\begin{array}{c}\text { Diameter }^{\mathrm{SiO}} \mathrm{O}_{2} \\
\mathrm{NP}^{\mathrm{c}}(\mathrm{nm})\end{array}$ \\
\hline PS5K10 & $-5.26 \pm 2.85$ & $-57.0 \pm 7.7$ & 23.9 & 0.425 & $39.1 \pm 1$ \\
$\mathrm{PS} 10 \mathrm{~K} 40$ & $-1.52 \pm 2.93$ & $-39.5 \pm 6.2$ & 20.7 & 0.242 & $37 \pm 1$ \\
$\mathrm{PS} 20 \mathrm{~K} 360$ & $-1.98 \pm 3.10$ & $-42.9 \pm 5.5$ & 23.9 & 0.078 & $37 \pm 1$ \\
$\mathrm{SiO}_{2} \mathrm{NPs}$ & $-3.52 \pm 3.50$ & -40 to $-50^{1}$ & 23.8 & 0.031 & not determined
\end{tabular}

${ }^{a}$ Hydrodynamic radius determined directly after the coating process with dynamic light scattering (DLS) using a Zetasizer Nano XS (Malvern); ${ }^{b}$ determined from the DLS experiment; ' diameter determined from TEM image analysis analyzing at least 50 nanoparticles. The sample was spotted on the grid directly after the coating process.
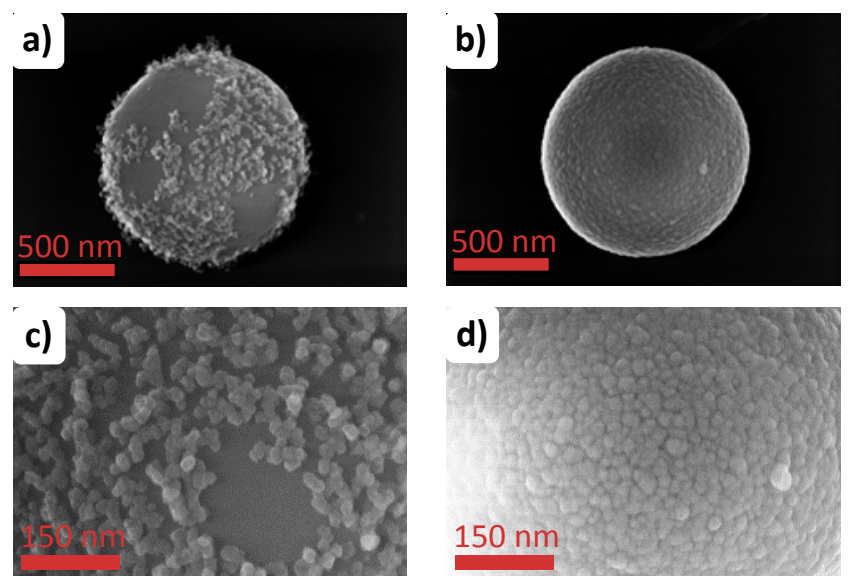

Figure S5: SEM images of $\mathrm{SiO}_{2} @ P S 360(a, c)$ and $\mathrm{SiO}_{2} @ \mathrm{PS} 10(\mathrm{~b}, \mathrm{~d})$ with lower amount of TEOS during the coating process $(0.5 \mathrm{~mL}$ instead of $1.5 \mathrm{~mL})$.

\footnotetext{
${ }^{1}$ Kim, K.-M.; Kim, H. M.; Lee, W.-J.; Lee, C.-W.; Kim, T.-i.; Lee, J.-K.; Jeong, J.; Paek, S.-M.; Oh, J.-M. Surface Treatment of Silica Nanoparticles for Stable and Charge-Controlled Colloidal Silica. Int. J. Nanomed. 2014, 9 (Suppl. 2), 29-40.
} 


\section{II.II Nitrogen adsorption/desorption and particle calculations}
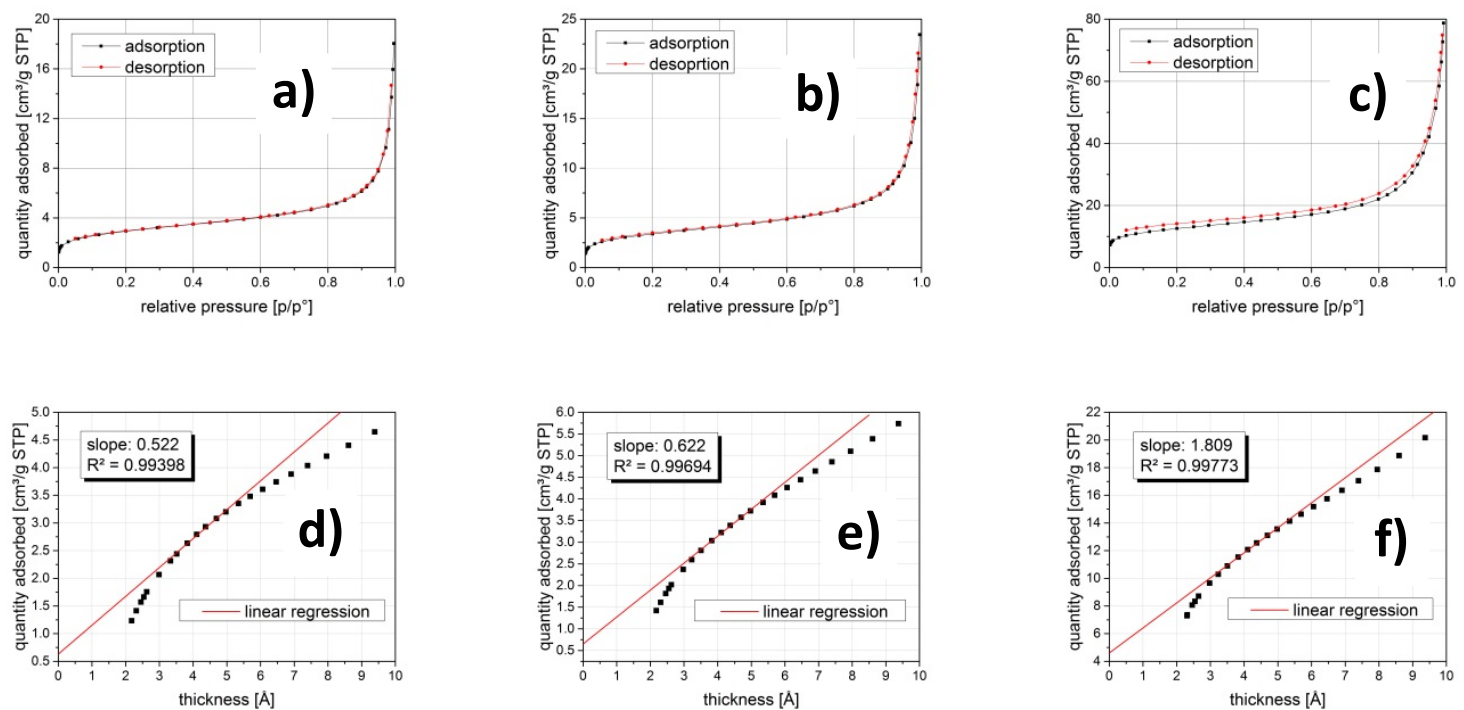

Eq. 1. $\quad t(\AA)=\sqrt{\frac{13.99}{0.034-\log \left(\frac{p}{p_{0}}\right)}}$ (Harkins and Jura)

Eq. 2. $S_{\text {ext }}=($ slope $\times 15.468)$

Figure S6. Nitrogen adsorption/desorption curves of (a) SiO $2_{2} @ P S 10$, (b) SiO $\mathrm{O}_{2} @ P S 40$ and (c) SiO $\mathrm{P}_{2} @ P S 360$. In all cases, isotherms of type II were observed with a discernible hysteresis for SiO $_{2} @ P S 360$. t-Plot of the nitrogen adsorption isotherm using Harkins and Jura correlation and the BET-adsorption data of (d) SiO $@$ @PS10, (e) $\mathrm{SiO}_{2} @ \mathrm{PS} 40$ and (f) $\mathrm{SiO}_{2} @ \mathrm{PS} 360$. To convert the slope into external surface area, the density conversion factor 0.0015468 was used.

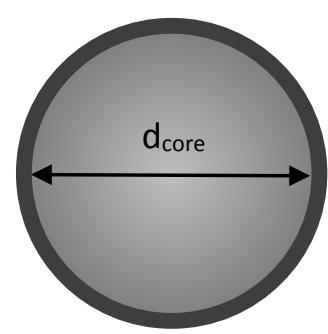

Eq. 3. $\quad V_{\text {core }}=\left(\frac{4}{3}\right) \times \pi \times\left(\frac{d}{2}\right)^{3}$

$\mathrm{d}=$ diameter $(\mathrm{nm})$

$\mathrm{V}=$ volume $\left(\mathrm{nm}^{3}\right)$

Eq. 4. $\quad m_{\text {core }}=\frac{V_{\text {core }}}{10^{18}} \times \rho_{P S}$

Eq. 5. $\quad m_{T G A}=1-\left(1 g *\left(\frac{\operatorname{SiO2}(\%)}{100)}\right)\right)$

$\mathrm{m}=$ mass $(\mathrm{mg})$

$\mathrm{m}_{\text {TGA }}=$ mass fraction of

Eq. 6. particles $\times g^{-1}=\frac{m_{T G A}}{m_{\text {core }}}$

$\rho_{\mathrm{PS}}=$ density $(\mathrm{PS})=1.05$ $(\mathrm{g} / \mathrm{mL})$

Figure S7. Calculation of particle numbers from TGA data, diameter as determined from T-SEM image analysis and average density of PS. 


\section{II.III Sedimentation, chemical surface characterization and assay performance}

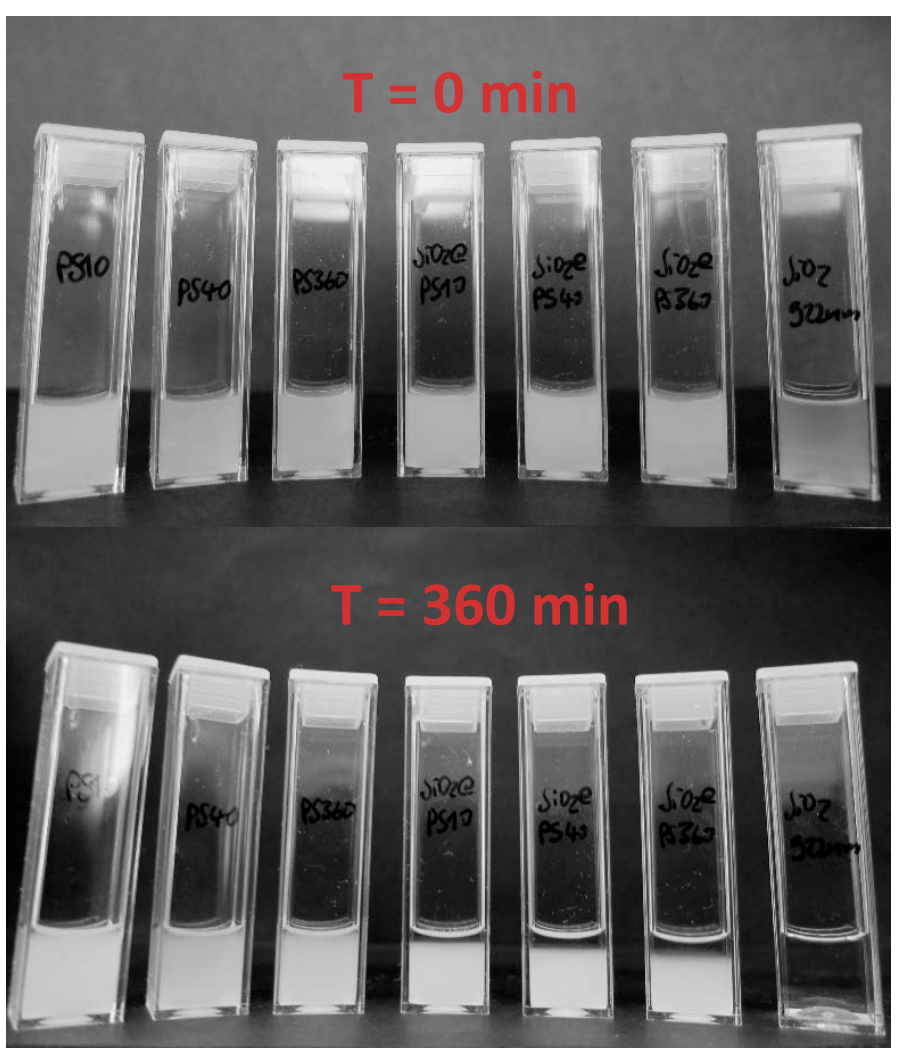

Figure S8. Temporal investigation of sedimentation behavior of the particles in aqueous dispersion $\mathbf{0 . 1 \%}$ w/v). Neat silica microparticles (922 $\pm 30 \mathrm{~nm}$, MicroParticles $\mathrm{GmbH}$, Germany) were used for comparison.

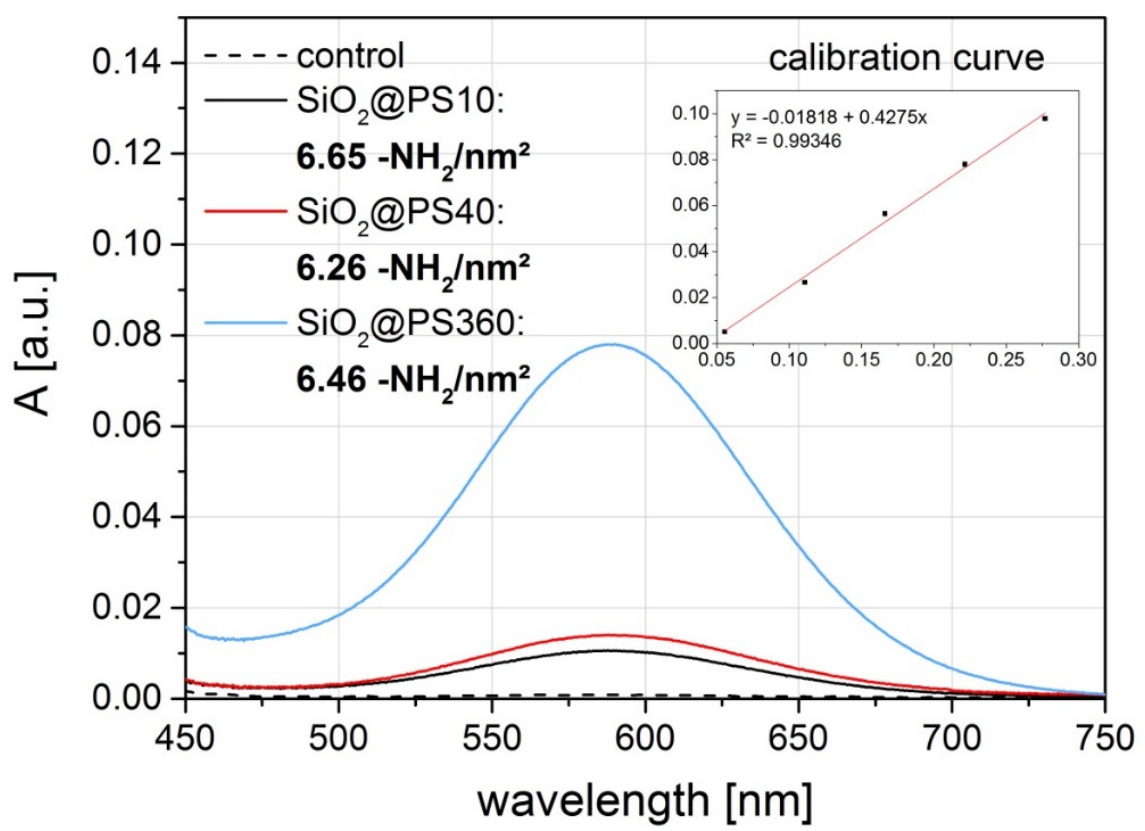

Figure S9. Absorption spectra of the supernatant after reaction with ninhydrin. The inset shows the calibration curve of ninhydrin test using pentylamine as a standard. 

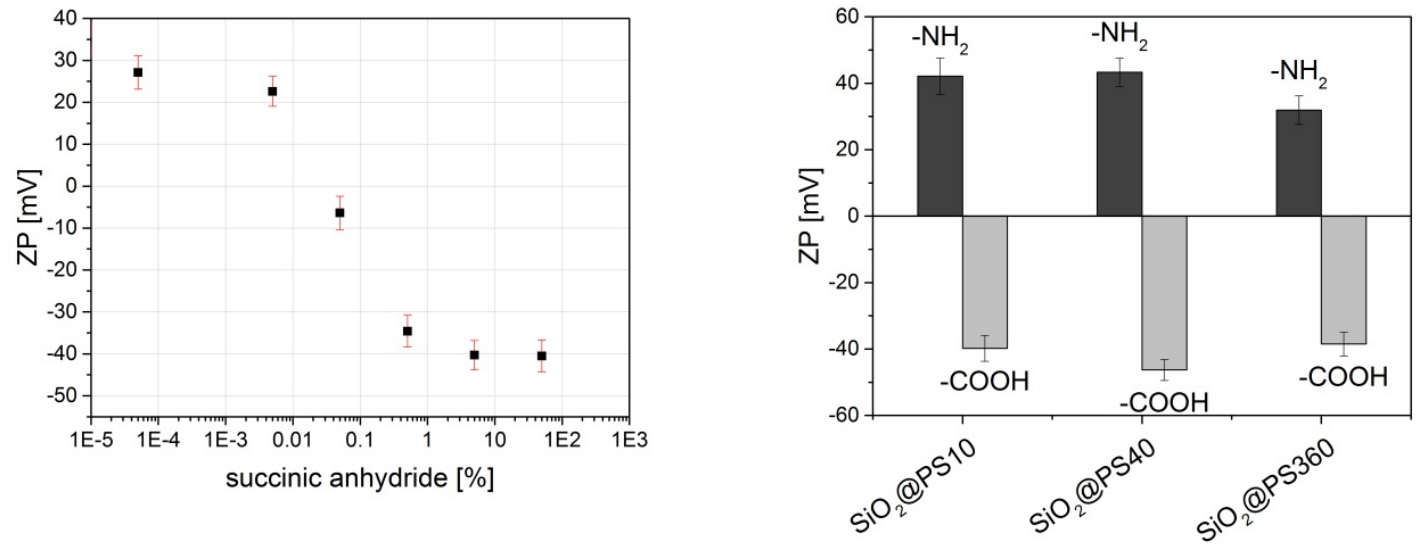

Figure S10. Left: Zeta potential measurements for carboxylated particles with different concentrations of succinic anhydride. Right: Zeta potentials of particles before $\left(-\mathrm{NH}_{2}\right)$ and after $(-\mathrm{COOH})$ carboxylation.
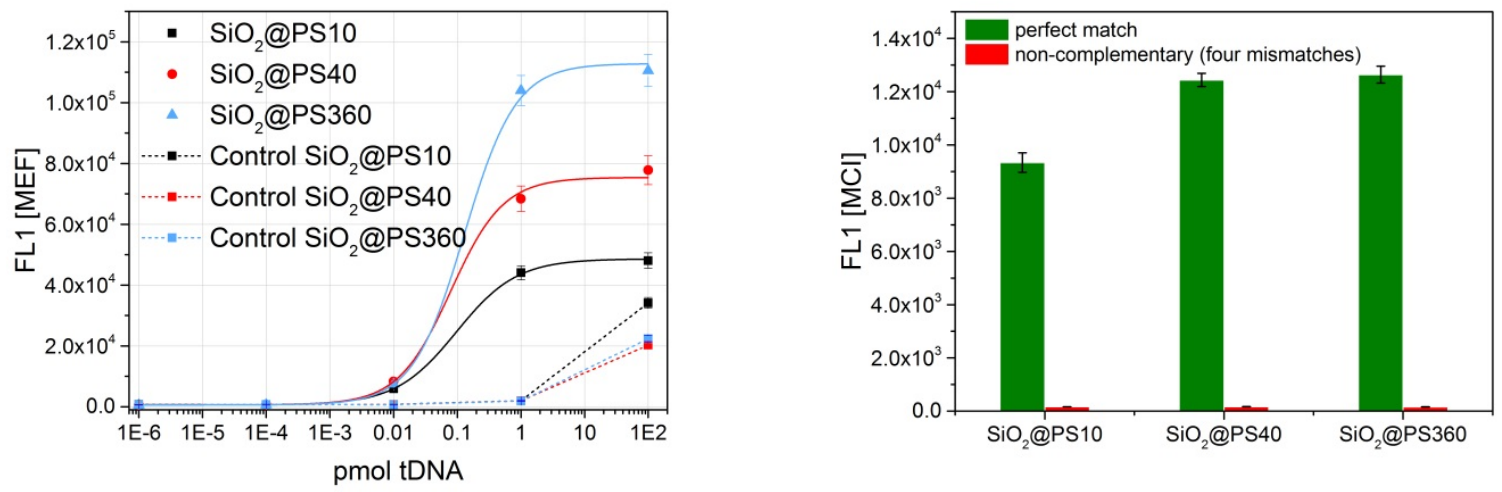

Figure S11. Left: Dose-response curve for the hybridization of complementary FAM-labeled t-DNA towards cDNA-modified and unmodified (control) core-shell beads. Right: Signal intensities in MCl with complementary and non-complementary (four adenosine units were exchanged by cytosine) t-DNA. 
II.IV Correlation plots for all cytometric measurements
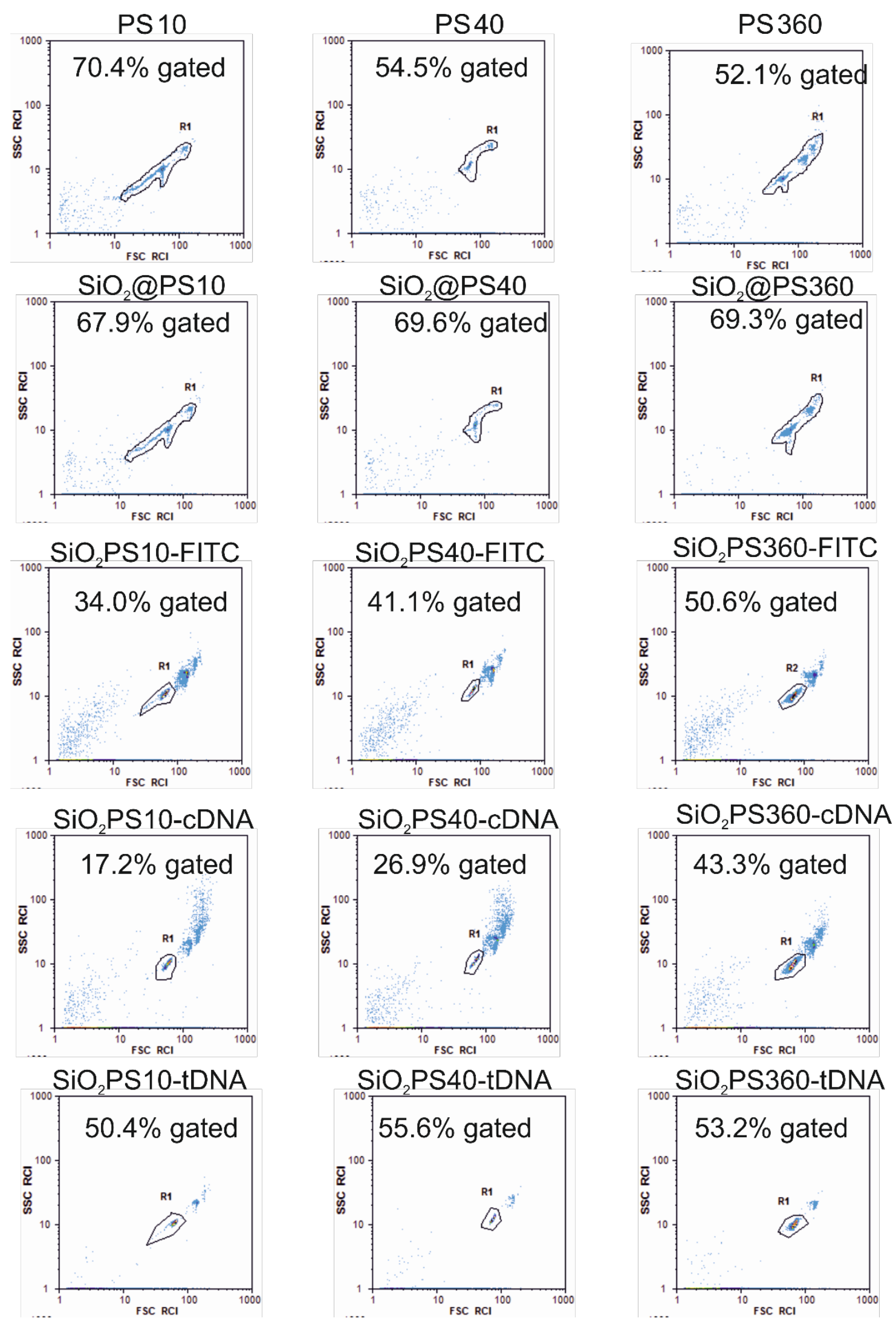

Figure S12. Correlation plots of FSC and SSC were used to set gates (R1). Relative numbers of events counted within the gates are shown in percent. “@” symbol omitted in some of the legends because of limited space. 